\title{
Is training of the lingual musculature necessary to treat orofacial functions?
}

Roberta Lopes de Castro Martinelli( ${ }^{(1)}$ Irene Queiroz Marchesan ${ }^{(1)}$ (1) CEFAC - Saúde e Educação, São Paulo,
São Paulo, Brasil.

Conflict of interests: Nonexistent

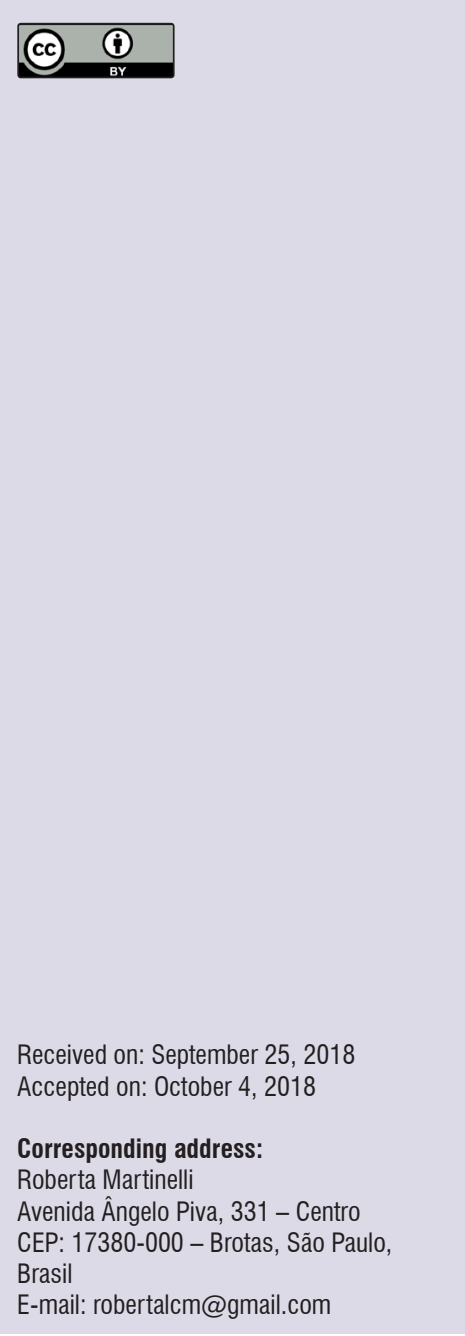

\section{Dear Editor-in-Chief,}

This letter aims at: a) discussing about the anatomy and physiology of the tongue, taking into consideration what the literature reports about the specificity of exercises, b) reflecting about the training of the lingual musculature during speech therapy, and c) incentivizing more research in this field.

The human tongue has a complex architecture consistent with its five crucial roles: breathing, sucking, swallowing, chewing, and speech. The tongue is ideally located to perform those orofacial functions, that is, it is located at the entrance to the gastrointestinal system and the respiratory system. The tongue is unique in the body as it is a deformable structure with no bones, joints, or air filled chambers, which nonetheless, must move about within the oral cavity ${ }^{1}$.

Although the human tongue plays a key role in performing vital functions, its structure and functions are not deeply understood ${ }^{2}$.

One of the main reasons for the lack of knowledge is that the tongue is part of a group of biological structures, including tentacles of cephalopod molluscs and the trunks of elephants. These structures have a constant volume and muscle groups arranged in several directions. Those characteristics make this structure particularly difficult to study3.

These structures, named muscular-hydrostats, have a remarkable diversity and complexity of movement. Since they are muscular organs that lack typical systems of skeletal support, they may produce bending, elongation, shortening, and torsion in any area or in multiple areas along its structure ${ }^{3}$.

Due to the dynamic support system, the tongue may perform an incredible variety of movements and adaptability during feeding or speech ${ }^{4}$.

The human tongue's myoarchitecture consists of a large array of variable aligned and extensively interwoven intrinsic and extrinsic muscle, which allow almost unlimited functional deformation ${ }^{5}$.

Gross tongue motion caused by the contraction of any individual muscle is dependent on the activity of surrounding muscles. Together those muscles produce a variety of intricate but well-controlled movements including tongue retraction, 
protrusion, dorsiflexion, ventroflexion, retroflexion, shortening, elongating, elevation, and depression².

Without any bony skeleton, the muscles themselves must provide the scaffolding upon which they can mechanically interact. The contraction of the vertical and transverse fibers is stabilized by the longitudinal fibers and vice-versa. Movement of the lateral aspect of the tongue requires support from the medial aspect. Similarly, movement of more distal segments of the tongue depends on support from the tongue base ${ }^{6}$.

Human tongue muscles are divided into two main groups: the extrinsic (genioglossus, styloglossus, and hyoglossus) and the intrinsic (superior longitudinal, inferior longitudinal, and the vertical and transverse) ${ }^{6}$. The extrinsic muscles have one attachment to the mandible or hyoid bone while the other end inserts within the tongue ${ }^{2}$. These muscles act more to change the position of the tongue within the oral cavity. The intrinsic muscles originate and insert within the tongue and have no bony attachments. These muscles are responsible for altering the shape of the tongue. Therefore, the intrinsic muscles of the tongue are not skeletal but striated because they do not originate and insert within any bone. However, the parts of the extrinsic muscles that enter into the tongue may be considered intrinsic muscles, which also make significant contributions to the shaping of the tongue ${ }^{2}$.

The intrinsic fibers are delicately merged with extrinsic muscles, modifying shape and position as well as functioning synergistically to constitute an almost unlimited number of physiological deformation ${ }^{5}$.

Two other interesting aspects of the tongue muscles are the fact that the muscle fiber orientation varies as the tongue changes its shape, and the effect of the contraction of any fiber groups on the body of the tongue are highly dependent on the activity of the rest of the fibers ${ }^{5,7}$.

Regarding the type of muscle fiber, in the tongue of adults there are a great number of type I muscle fibers, which slow down the movements and are more resistant to fatigue. Slow fibers are involved in activities requiring precise control of low forces, indicating that the human tongue has specialized for these movements ${ }^{6}$.

However, the proportion of type I muscle fibers distributed in the three parts of the tongue (base, body and blade) is not homogeneous nor is it homogeneous in the same muscle, in different muscles, or in different specimens, and nor in adverse health condition ${ }^{6}$.

In the superior longitudinal muscle, for example, there is a greater number of slow fibers in the base and body of the tongue, whereas in the blade, there is a greater number of fast fibers, which allow the blade to perform delicate and fast movements by changing its shape ${ }^{6}$.

Taken together, these unique properties of the tongue suggest that any one-movement goal maybe accomplished by a variety of muscle activation patterns. Moreover, any given muscle fiber may be recruited for a number of movement goals. Thus, effects of training may be less specific than observed for skeletal muscle groups $^{8}$.

A well-established principle in muscle training is specificity, which has been observed for numerous aspects of muscle training, including strength, endurance, power, and speed $^{8}$. It is not well known how training specificity by means of exercises may be manifest in the muscle group of the tongue ${ }^{8}$.

Strength training causes the muscle fibers type II to adapt. To date no studies have examined the adaptation of muscle fibers in the tongue. Lingual strength may be increased with a variety of exercise protocols; however, the effects of the detraining may also be observed when training is discontinued ${ }^{9}$.

Resistance training causes the type I muscle fibers, which are resistant to fatigue, to adapt. Although the anterior part of the tongue has a relative shortage of type I fibers $(21 \%)$ they consist of more than half of the fibers in the posterior part of the tongue ${ }^{10}$.

The velocity of contraction is subject to the specificity of training with exercises protocols performed with fast movements ${ }^{11,12}$. A study demonstrated that speed training for tongue muscles has no effect ${ }^{8}$.

Finally, high speed and moderate resistance exercises cause the type IIA muscle fibers to adapt ${ }^{8}$. This type of fibers is quite rare in the tongue ${ }^{10}$.

Thus, the morphology and biomechanical properties of the tongue differ substantially from the skeletal muscles, which leads us to reflect whether muscle training should follow the same principle of the exercise programs proposed for the skeletal striated muscle and how specificity training may manifest itself in this muscle group.

Based on the previous considerations, we question whether tongue training should be applied, or the functions should be addressed directly; whether the exercises can mimic all the movements performed by the tongue in the orofacial functions.

Further studies are needed to clarify the anatomy and physiology of the tongue as well as the impact of muscle training on the tongue. 
We expect that the aspects discussed in this article may stimulate future studies, thus contributing to the understanding of both the anatomy and the specificity of the tongue muscle training, and enriching the Speech-Language Pathology practice.

\section{REFERENCES}

1. Stone M, Woo J, Lee J, Poole $T$, Seagraves $A$, Chung $M$ et al. Structure and variability in human tongue muscle anatomy. Comput Methods Biomech Biomed Eng Imaging Vis. 2018;6(5):499-507.

2. Sanders I, Mu L. A three-dimensional atlas of human tongue muscles. Anat Rec (Hoboken). 2013;296(7):1102-1.

3. Kier WM, Smith KK. Tongues, tentacles and trunks: the biomechanics of movement in muscular hydrostats. Zool J Linn Soc. 1985; 83:307-24.

4. Stavness I, Sánchez CA, Lloyd J, Ho A, Wang J, Fels $S$ et al. Unified skinning of rigid and deformable models for anatomical simulations. Technical Briefs on - SIGGRAPH ASIA, 2014.

5. Gaige TA, Benner T, Wang R, Wedeen VJ, Gilbert RJ. Three dimensional myoarchitecture of the human tongue determined in vivo by diffusion tensor imaging with tractography. J Magn Reson Imaging. 2007;26(3):654-61.

6. Sanders I, Mu L, Amirali A, Su H, Sobotka $\mathrm{S}$. The human tongue slows down to speak: muscle fibers of the human tongue. Anat Rec. 2013;296(10):1615-27.

7. Elsaid $\mathrm{NMH}$, Stone M, Roys S, Gullapalli RP, Prince JL, Zhuo J. Diffusion spectrum imaging tractography of the human tongue. Annals 25th Annual Meeting ISMRM. 2017.

8. Clark HM. Specificity of training in the lingual musculature. J Speech Lang Hear Res. 2012;55(2):657-67.

9. Clark HM, O'Brien K, Calleja A, Corrie SN. Effects of directional exercise on lingual strength. J Speech Lang Hear Res. 2009;52(4):1034-47.

10. Stål $P$, Marklund $S$, Thornell LE, De Paul R, Eriksson PO. Fibre composition of human intrinsic tongue muscles. Cells Tissues Organs. 2003;173(3):147-61.

11. Behm DG, Sale DG. Velocity specificity of resistance training. Sports Med. 1993;15(6):374-88.

12. Pereira Ml, Gomes PS. Movement velocity in resistance training. Sports Med. 2003;33(6):427-38. 